Rabaska

Revue d'ethnologie de l'Amérique française

\title{
La collaboration entre Adélard Lambert et Marius Barbeau
}

\section{Danielle Martineau}

Volume 13, 2015

Présence de Marius Barbeau : l'invention du terrain en Amérique française. Autour d'un legs centenaire (1914-2014)

URI : https://id.erudit.org/iderudit/1033760ar

DOI : https://doi.org/10.7202/1033760ar

Aller au sommaire du numéro

Éditeur(s)

Société québécoise d'ethnologie

ISSN

1703-7433 (imprimé)

1916-7350 (numérique)

Découvrir la revue

Citer cet article

Martineau, D. (2015). La collaboration entre Adélard Lambert et Marius

Barbeau. Rabaska, 13, 179-187. https://doi.org/10.7202/1033760ar d'utilisation que vous pouvez consulter en ligne.

https://apropos.erudit.org/fr/usagers/politique-dutilisation/ 


\section{La collaboration entre Adélard Lambert et Marius Barbeau}

Danielle Martineau

Médiatrice du patrimoine vivant, Saint-Alphonse-Rodriguez

Les travaux du projet « L'héritage culturel d'Adélard Lambert », dont m'a chargée la Municipalité régionale de comté (MRC) de D’Autray en 2014 dans Lanaudière, sont soutenus par le ministère de la Culture et des communications du Québec (MCCQ), par le Conseil des arts du Canada et par la MRC de D'Autray. Il s'agit d'une mise en valeur qui vise à obtenir des résultats durables et pertinents dans l'optique de l'établissement d'un pôle culturel de qualité. Cette action permet de sensibiliser tout un bassin - élus, citoyens, familles, artistes, porteurs de tradition - à l'importance de la reconnaissance du patrimoine oral, ce patrimoine si vivant dans la communauté de D'Autray. Cet exposé entend résumer le cheminement et les défis de cette expérience de médiation culturelle en soulignant particulièrement les enseignements qui découlent de la collaboration fructueuse entre Adélard Lambert et Marius Barbeau dans cette relation unique et exemplaire.

\section{Cheminement du projet}

Adélard Lambert, collaborateur de Marius Barbeau, est né à Saint-Cuthbert, situé sur le territoire de la MRC de D'Autray. J'ai pris connaissance de cette collaboration en 1999, alors que je réalisais une recherche subventionnée par le Conseil des arts du Canada, sur les 4000 premiers enregistrements sonores du fonds Barbeau.

Les principales particularités de la collection Lambert sont d'abord qu'il s'agit d'un portrait familial des pratiques de chansons, jeux, danses chantées et contes, contrairement à l'approche habituelle de recherche de contenu auprès d'informateurs et d'informatrices isolés du contexte de pratique familiale ou sociale. M. Lambert a d'ailleurs réuni ses frères et sœurs vivants pour l'aider à recenser tous leurs souvenirs, qui constituent la majorité des éléments de la collection. Ensuite, cette collection fournit un grand nombre de pratiques 


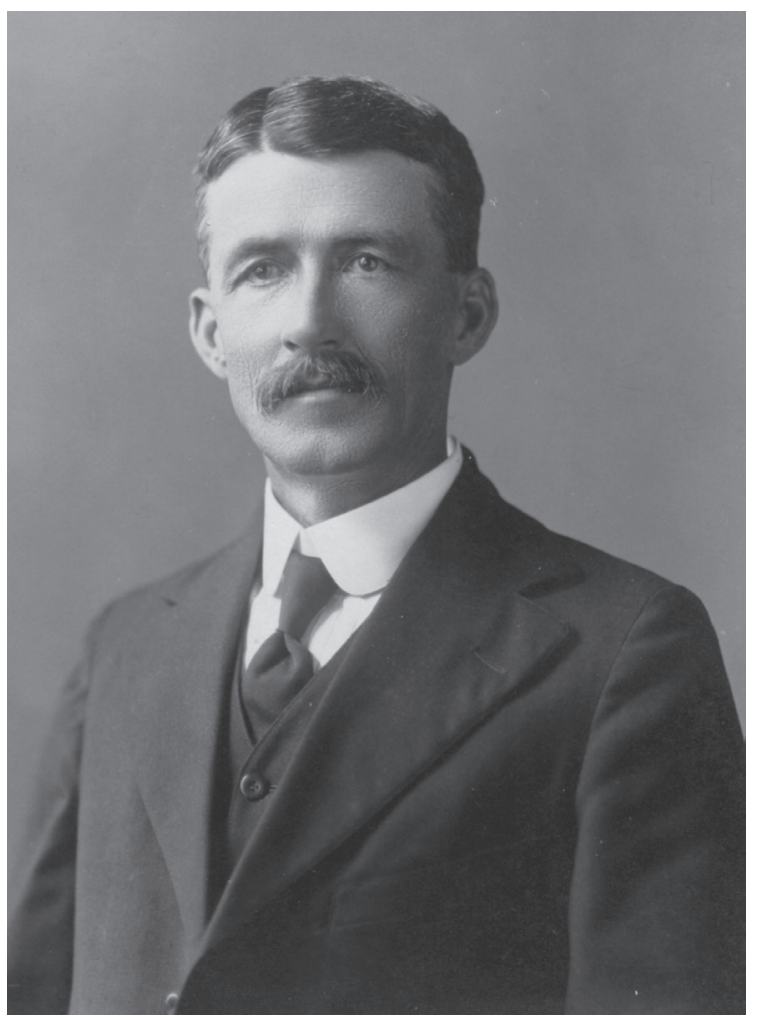

Adélard Lambert, avant 1935

Source : Collection Madeleine Chabot, petite-fille d'A. Lambert

traditionnelles concernant l'enfance : berceuses, jeux, formulettes, danses chantées et contes.

En tant qu'artiste et médiatrice du patrimoine vivant résidant dans Lanaudière depuis 1991, j'ai observé et étudié de très près la dynamique familiale à l'œuvre dans la pratique et la transmission de ces traditions. Une partie des connaissances sur la transmission familiale provient d'une recherche de deux ans auprès de soixante-dix familles du territoire qui a donné lieu à la publication du livre On a du plaisir nous autres ${ }^{1}$.

Depuis la mise en œuvre en 2012 de la loi 82 sur le patrimoine culturel, on reconnait finalement la valeur culturelle des pratiques traditionnelles, maintenant désignées patrimoine immatériel au MCCQ, nommées depuis plus de vingt ans patrimoine vivant par les travailleurs et organismes du milieu

1. Danielle Martineau et Lisan Hubert, On a du plaisir nous autres. Traditions orales de Lanaudière, livre-disque, [Saint-Jean-de-Matha], Centre régional d'animation du patrimoine oral de Lanaudière (CRAPO), 2004, [10]-198 p. 
depuis le colloque «Événement national sur les arts et les traditions populaires ", à Jouvence, en 1984. Suite à l'adoption de la loi 82, un programme de soutien à des projets de sauvegarde et de mise en valeur a alors été créé, mais il a été éliminé en septembre 2014.

La MrC de D'Autray, par l'intermédiaire de son agente culturelle à qui j'avais signalé l'existence de la collection Lambert, a décidé d'innover comme institution et a obtenu un financement pour réaliser la première phase d'un projet de mise en valeur de l'héritage culturel d'Adélard Lambert. Et elle m'a confié la charge de le diriger.

\section{L'héritage culturel d'Adélard Lambert}

Le projet visait à mettre en valeur un formidable héritage culturel provenant du plus ancien fonds d'archives manuscrites et sonores, réalisé par un collectionneur et folkloriste originaire de Lanaudière. La collection inédite d'Adélard Lambert (1867-1946), comprend près de 700 faits de tradition orale : chansons, contes, danses chantées et jeux de l'enfance, issus en majeure partie de son héritage familial. M. Lambert a également été un auteur et un bibliophile. Il a collectionné 4000 ouvrages témoignant de la présence française en Amérique. Vers 1919, l'Association canado-américaine, toujours active à Manchester, New-Hampshire, fait l'acquisition de son imposante collection. Elle sert de base au centre de documentation de l'Association.

Grâce à la collaboration entre M. Lambert et Marius Barbeau, les manuscrits et enregistrements sur rouleaux de cire sont conservés aux archives du Musée canadien de l'histoire à Gatineau, dans le fonds Marius Barbeau.

L'objectif global du projet est que l'ensemble de la population de la MrC de D'Autray puisse se réapproprier son patrimoine culturel immatériel par une meilleure connaissance du personnage, du contenu de sa collection et par sa transmission. Dans ce contexte, le projet accompagne également le processus légal municipal d'identification d'Adélard Lambert au registre du patrimoine culturel du McCQ en tant que personnage historique signifiant pour la municipalité de Saint-Cuthbert en 2015.

Voici un bref aperçu des activités réalisées en 2014. Les documents manuscrits et sonores de la collection Lambert ont été analysés dans le but d'en évaluer la valeur patrimoniale. Un comité citoyen porteur du projet a été mis en place et le plan de transmission y a été élaboré en identifiant tous les secteurs de la population concernés par le contenu de la collection : famille, culture, loisir, éducation, milieu communautaire, milieu politique et développement durable. Une recherche dans la communauté de D'Autray, a permis de valider l'actualité de la pratique des faits de folklore consignés dans le fonds Lambert : concordance, évolution et rupture. Onze activités de sensibilisation, dont cinq intergénérationnelles, onze rencontres de transmission 
de jeux et de jeux chantés au sein des activités des camps de jour d'été et six ateliers intergénérationnels de transmission ont été réalisés sur l'ensemble du territoire. Grâce à la collaboration du Musée canadien de l'histoire, la MrC a créé un lieu de consultation de la collection Lambert, dans le but de rapprocher les archives des citoyens. Les descendants vivants de M. Lambert ont été trouvés et avisés de cette démarche.

De manière plus spécifique, la municipalité de Saint-Cuthbert, lieu de naissance d'Adélard Lambert, a inséré plusieurs activités supplémentaires de transmission à l'école et pour toutes les générations dans le cadre des célébrations du $250^{\mathrm{e}}$ anniversaire de la municipalité de Saint-Cuthbert en 2015. Le journal citoyen de Saint-Cuthbert, le Ça $m$ 'Chicotte édité quatre fois par année et disponible en ligne, fait état des développements de ce projet de mise en valeur.

Toutes ces actions ont été produites en lien avec les objectifs de la politique culturelle de la MRC de D'Autray, plus particulièrement le renforcement de l'identité régionale par la sensibilisation à la richesse collective reflétée de manière éloquente par les travaux de M. Lambert.

\section{Les défis}

\section{L'état de la collection}

Dans un premier temps, il fallait donc recueillir le plus grand nombre de documents issus des travaux réalisés par M. Lambert afin d'en faire l'analyse et le traitement - les budgets concernant le traitement de la documentation ont été soustraits du financement par le MccQ. Malgré l'absence de traitement complet, la collection Lambert est toutefois accessible à la population depuis septembre 2014, dans le même état de présentation des dossiers qu'aux archives du Musée canadien de l'histoire $(\mathrm{McH})$ grâce à une entente entre le $\mathrm{MCH}$ et la MrC qui permet la consultation des documents à la MrC de D'Autray, dans les mêmes conditions que celles des archives du McH à Gatineau. Cette entente innovatrice répond à l'objectif de rendre plus accessible à la population la documentation recueillie par M. Lambert, ce que le musée souhaite si ardemment.

Les documents disponibles nous révèlent un nombre impressionnant de savoirs, principalement détenus par la mère d'Adélard Lambert, Léocadie Rinfret dit Malouin, originaire de Maskinongé, qui constituent la majorité des entrées. M. Barbeau a guidé, par une approche de soutien exemplaire, M. Lambert dans sa collecte des faits de sorte qu'il les a bien documentés en précisant de qui il détient les savoirs, à quel âge il est entré en contact avec ces connaissances, l'âge, l'année de naissance et la provenance des « informateurs »; de plus, il a établi une numérotation des documents et une 
concordance entre les numérotations de documents manuscrits et sonores s'il y a lieu.

Celle collection est accompagnée d'une correspondance assidue entre messieurs Barbeau et Lambert de 1921 à 1944, les deux hommes ne s'étant pratiquement jamais rencontrés physiquement. $M$. Barbeau avait prédéterminé un système de numérotation réservé à ce collaborateur, mais $\mathrm{M}$. Lambert lui a fourni tellement d'entrées que la numérotation s'en est trouvée problématique.

La documentation manuscrite a été fragmentée. En effet, M. Barbeau, à l'époque où il quittait l'institution muséale d'Ottawa et devenait actif à l'Université Laval a apporté avec lui une partie des documents de cette collection, qui se sont retrouvés aux Archives de folklore de l'Université Laval. En prenant connaissance de l'ensemble des documents manuscrits, j'ai pu constater que le système de numérotation a déraillé vers les deux tiers de la collection et qu'on a pallié par une double numérotation qui n'aide pas à s'y retrouver ; que certains éléments de la collection se sont perdus, en partie dans les déplacements des manuscrits d'Ottawa à Québec ; et que, sur certains documents pour une entrée donnée, on peut lire la description d'un jeu, aussi d'une comptine ou d'une formulette, ce qui devrait être réparti dans des entrées distinctes.

Par ailleurs, plusieurs entrées sonores sont encore audibles, mais l'état des enregistrements sonores nous montre qu'une partie d'entre elles sont manquantes, inaudibles ou numérotées en double ; que les transferts de support présentent des erreurs de vitesse ; que tous les documents sonores ne sont pas encore numérisés.

La mission première de conservation a été remplie au sein des institutions archivistiques, mais peu de personnes y ont pris en charge la valorisation de ce type de collection après Marius Barbeau, souvent par manque de financement et de personnel.

En somme, la majorité de la collection manuscrite a été préservée, mais, malgré toutes les bonnes intentions, des éléments manquent et quelques-uns sont en plus ou moins bon état. Il est bon de savoir que les supérieurs de M. Barbeau n'acceptaient pas de bon gré les travaux concernant les traditions canadiennes-françaises et que, pendant de nombreuses années, il a dû faire preuve de détermination pour les préserver et les publier.

\section{Les nouveaux partenaires institutionnels : MRC et municipalités}

Il est très nouveau qu'une instance comme une MrC s'engage dans le domaine du patrimoine immatériel. La rigueur des travaux dans ce domaine est actuellement mal connue et évaluée. Aussi le médiateur doit-il donner beaucoup d'information dans un cadre limité par les sommes attribuées au projet. La 
compréhension se bâtit en expliquant pourquoi et comment on choisit ses interventions. Mon parcours professionnel m'a heureusement mise en contact avec les outils créés par l'approche de la pédagogie culturelle, développée en Ontario francophone. Ce modèle d'intervention très efficace peut se résumer comme suit : accompagner les prises de conscience, accompagner les décisions et accompagner les prises en charge.

Bref, tout reste à bâtir en ce qui concerne les stratégies de mise en valeur du patrimoine immatériel dans ces institutions municipales, maintenant désignées par le MCcQ comme les maîtres d'œuvre de la sauvegarde du patrimoine immatériel. C'est pourquoi j'ai conçu des formations de base sur le terrain à cet effet.

\section{Adélard Lambert, un personnage inconnu des Québécois}

Comment réaliser une démarche publique dans le monde d'aujourd'hui avec un sujet aussi inconnu? La MRC a pris la responsabilité, selon ses moyens, du plan de promotion des activités : conférence de presse, communiqués, outils publicitaires, etc.

\section{Les avantages}

Comme M. Lambert est né à Saint-Cuthbert, il est rapidement devenu un « héros » local et l'adhésion des élus et des partenaires a été unanime et immédiate. Dans son village natal, Adélard Lambert est un personnage dont on évoque le nom avec fierté et sympathie, sans être totalement conscient de la valeur de son travail. Les rencontres citoyennes de sensibilisation ont tout de même confirmé que ceux et celles qui réalisent la détermination et la richesse du parcours et des travaux de M. Lambert réagissent avec étonnement et admiration.

\section{Les inconvénients}

L'espace médiatique réservé à la culture est très restreint en région. Un journal régional hebdomadaire accueille les communiqués, et la présence des informations culturelles est actuellement limitée au portail de la MrC. Compte tenu de ces maigres moyens, il est impossible de positionner les activités de mise en valeur du projet de manière signifiante au sein de la compétition féroce des innombrables informations circulant sur les plateformes numériques. De plus, comme les programmes de soutien du MccQ ont été coupés pour l'année 2015, il faudra trouver d'autres stratégies pour poursuivre le travail de mise en valeur. 


\section{Les enseignements}

\section{L'obsession d'Adélard Lambert pour la publication}

En parcourant la correspondance entre messieurs Barbeau et Lambert, il est clair que celui-ci acheminait sa documentation à M. Barbeau en vue de la publication. Malgré une scolarisation écourtée, M. Lambert avait la forte intuition que le livre allait sauver de l'oubli les connaissances si précieuses qu'il chérissait. La réponse de $\mathrm{M}$. Barbeau à ces demandes répétées a été exemplaire dans la limite de ses moyens, intégrant plusieurs de ses documents dans des numéros du Journal of American Folklore, dans les pages pour enfants de différents journaux - Le Droit d'Ottawa, La Patrie et La Presse de Montréal -, et dans ses ouvrages autonomes : le Romancero du Canada, Dansons à la ronde, Les enfants disent. Cette vaste diffusion montre aussi la volonté de Marius Barbeau de mettre ces connaissances à la portée de tous.

M. Lambert suivait avec assiduité le cheminement et les résultats des publications, obsédé par son souci de pérenniser les richesses culturelles héritées de sa mère et de son entourage. Par ailleurs, nous avons appris récemment que sa famille et sa descendance n'ont jamais réalisé l'existence de cette collaboration, ce qui peut porter à croire qu'il se sentait rassuré par les encouragements et réalisations de M. Barbeau, plus que par son entourage immédiat.

De son côté, M. Barbeau fournissait des renseignements précieux sur la provenance des répertoires recueillis par M. Lambert. Ce traitement permet aujourd'hui de donner une valeur ajoutée à la collection Lambert et de présenter aux preneurs de décisions, en toute objectivité et preuves à l'appui, sa grande valeur patrimoniale.

\section{La réalité de la chanson de tradition}

La documentation sur la chanson traditionnelle réalisée par Marius Barbeau offre un champ encore fertile d'études sur cette pratique traditionnelle. Il s'est intéressé à la provenance des textes, en concordance avec les disponibilités de son époque. Possédant les bases de la transcription musicale, il a préparé de nombreuses partitions musicales, certaines ayant servi dans ses publications diverses. Cette compétence en écriture musicale devrait par ailleurs être inhérente à quiconque analyse la chanson traditionnelle. Il s'est aussi appliqué à comprendre et à exposer les particularités musicales des chansons, ses modes, ses rythmes, avec la collaboration d'ethnomusicologues réputés. On sait que le système d'écriture musicale occidental avec portées, barres de mesures, etc., est imparfait pour refléter avec exactitude la modalité et les rythmes ou non rythmes présents dans ce type de répertoire. La chanson traditionnelle comporte aussi ce que Jean-Michel Guilcher appelle le mouvement : 
plusieurs des chansons en laisse sont ou ont été des danses et comportent donc des rythmiques associées aux danses rondes qui leur correspondent.

À cet effet, un extrait d'une lettre de M. Lambert concerne directement cet aspect de la chanson à propos des « danses rondes et jeux d'enfants » :

M. Barbeau,

Il serait à-propos je crois, si vous veniez à publier un volume renfermant danses rondes et jeux d'enfants, d'y faire une introduction dans ce sens, dont je veux vous exposer mon idée dans quelques lignes, satisfait que vous pourriez y broder quelque chose de très supérieur.

On sait, qu'à venir à quelques années, il n'était nullement fait mention ici au Canada parmi nos jeunes gens, de jeux de « balle au champ » de « hokey », de « tenis »... etc. Alors, toute l'attention de nos jeunes était concentrée sur les jeux et danses rondes qui s'en vont vers l'oubli aujourd'hui. Même quelquesuns des vieux m'ont laissé entendre et assuré qu'un nombre considérable de nos chansons populaires telles que : En roulant ma boule ou Marianne s'en va-t-au moulin... etc. étaient des danses rondes très en vogue dans les premiers temps de la colonie. Et ce serait une des raisons pour laquelle ces vieux chants populaires se seraient mieux que bien d'autres conservés jusqu'à nous.

Ce document témoigne en effet que la mémoire du corps est plus ancrée que la mémoire du cerveau uniquement. Mes années d'observations sur le terrain confirment que les chansons en laisse, dites "à répondre » font encore l'objet d'une pratique fervente dans Lanaudière. Ce qui expose une autre dimension de la chanson traditionnelle beaucoup moins étudiée : la pratique des veillées de chansons où l'objectif est la transe, si nécessaire et libératrice des lourdeurs du quotidien et encore identifiée comme telle par ceux qui la vivent. Cet état recherché fait partie depuis des millénaires de la pratique de ce type de répertoire chansonnier, et ce dans toutes les traditions chantées sur la planète. Pour ma part, c'est une qualité et une « compétence » reliée à la chanson traditionnelle qui lui accorde une valeur patrimoniale indubitable. C'est ici pour moi que le mot transe-mission prend tout son sens.

\section{Connaissance et reconnaissance}

Dans cette étude de cas visant la mise en valeur d'un héritage culturel, il est nécessaire de prendre acte des obstacles politiques et religieux majeurs au traitement et à la publication des travaux de M. Barbeau au Québec et, par conséquent, des données recueillies par M. Lambert. Les efforts de M. Barbeau pour la publication ont donné des résultats éloquents par la qualité et par le nombre, tant par les publications en français dans le Journal of American Folklore que dans d'autres publications à l'extérieur du Québec, en français et en anglais. 
Il ressort de manière claire que $M$. Barbeau a également eu le souci d'être à l'écoute, d'outiller, d'encourager et de remercier M. Lambert pour sa contribution. L'enseignement magistral qui en découle est qu'il faut toujours se soucier des personnes qui communiquent leurs connaissances aux travailleurs des institutions dans notre domaine. Sans le souci d'inclure la reconnaissance dans ses actions, on perd un moyen très fort d'aider les porteurs de tradition et leur entourage à mesurer la valeur culturelle de leurs connaissances. C'est ainsi que l'éthique de travail d'un médiateur du patrimoine vivant est basée sur les valeurs de partage et de reconnaissance. Ces valeurs procurent une série d'autres valeurs comme la fierté, le sentiment d'appartenance et la conscience de la capacité d'agir pour prendre en charge la vitalité d'une tradition dans sa famille ou dans son entourage.

Dans un milieu familial, la médiation permet de discuter des motivations et des méthodes de transmission. Ces échanges, où on nomme tout simplement les valeurs en jeu, offrent des outils importants pour opérer une intervention plus adéquate. On peut maintenant observer dans Lanaudière, depuis quelques années, que les interventions en patrimoine vivant guidées par les valeurs de partage et de reconnaissance obtiennent un plus grand succès auprès de l'ensemble des citoyens. Cette quête de sens est le moteur qui nourrit le choix des actions, leur mise en œuvre et la manière la plus appropriée d'accompagner et d'intervenir auprès du milieu. 